\title{
A Study on Developing Pico Propeller Turbine for Low Head Micro Hydropower Plants in Nepal
}

\author{
Pradhumna Adhikari, Umesh Budhathoki, \\ Shiva Raj Timilsina, Saurav Manandhar, Tri Ratna Bajracharya \\ Energy Systems Planning and Analysis Unit, Centre for Energy Studies, Institute of Engineering, \\ Tribhuvan University, Nepal \\ Corresponding Email: atpradhumna@gmail.com
}

\begin{abstract}
Most of the turbines used in Nepal are medium or high head turbines. These types of turbines are efficient but limited for rivers and streams in the mountain and hilly region which have considerably high head. Low head turbines should be used in the plain region if energy is to be extracted from the water sources there. This helps in the rural electrification and decentralized units in community, reducing the cost of construction of national grid and also to its dependency, in already aggravated crisis situation.

There are good turbine designs for medium to high heads but traditional designs for heads under about $5 \mathrm{~m}$ (i.e. cross flow turbine and waterwheel) are slow running, requiring substantial speed increase to drive an AC generator. Propeller turbines have a higher running speed but the airfoil blades are normally too complicated for micro hydro installations. Therefore, the open volute propeller turbine with constant thickness blades was ventured as possible solution. Such type of propeller turbine is designed to operate at low inlet head and high suction head. This enables the exclusion of closed spiral casing. Also, the constant thickness blades enable the use of forging process instead of casting of complex airfoil blades. This leads to considerable reduction in manufacturing cost and complexity.

A $1 \mathrm{~kW}$ prototype was designed and scale down model of $185 \mathrm{~W}$ was fabricated and tested. The runner consisted of five blades of $4 \mathrm{~mm}$ thickness with camber and twist. The runaway speed of $1058 \mathrm{rpm}$ was attained at design flow rate of $25 \mathrm{l} / \mathrm{s}$. At full load the efficiency of model was found to be about 57\%. Applying scaling effects the expected efficiency of the prototype was estimated to be about $60 \%$.
\end{abstract}

\section{Problem / Background}

Due to the incompetent governance and lack of fund and technology, Nepal has been far behind in the production of hydroelectricity even though it has huge potential. Due to the unavailability of the national grid all over the country, many villages have not been electrified yet. Their electrification through the national grid is very expensive for our poor country. So, micro hydro plant is one of the best ideas for electrification there.

The hilly and the mountainous regions of Nepal have streams and rivers with high head, so most of the research and study works has been limited to the development of the efficient high head turbines in these regions. Enough study and research has not been done for the development of the turbines and micro hydro plants for the Terai region where the head is considerably low but the discharge is quite high. Until now, we have been discarding the huge water potential of the Terai. 
The use of the propeller turbine can be a good idea for harnessing the water potential in the Terai region. Micro hydro plants are easy to construct and they don't need huge amount of money for their construction and operation. Use of pico-propeller turbine in the micro hydro plants further reduces the cost and technical difficulty as this turbine can be locally manufactured. Its installation and fabrication cost is quite low. Propeller turbine is also suitable for the streams with high silt load.

\subsection{Pico-hydro in Nepal}

A majority of rural household in Nepal is not connected to the national grid, so they are compelled to seek alternative source of electricity. Many mini and micro hydropower plants have addressed this problem. However those communities which are not in the vicinity of potentially large water resources have been deprived of electricity. For these small and remote communities, pico-hydro can be the best energy solution.

At present, for small communities, peltric sets are available in pico-hydro range (below $5 \mathrm{~kW}$ ). As compact fluorescent light (CFL) technology has become cheaper and more affordable for villagers, a smaller amount of power generated can go a lot further in terms of lighting more households. $1 \mathrm{~kW}$ of power can provide over $100 \mathrm{CFL}$ bulbs (9W) for a community. So, picohydro can be very beneficial smaller villages that cannot afford a larger hydropower project.

In most cases, in Nepal, pico-hydro projects (peltric sets) use low flow rate and high head (40m). Unfortunately, many smaller villages do not have suitable sites for peltric set units. However, these villages do have existing irrigation canals. Also many hill communities use traditional water mills. These canals and mills can be suitable sites for pico-hydro. For these types of situations, propeller or cross flow turbines that run at high flow rate and low head can be applicable. So far the propeller type pico-hydro is under-utilized technology in Nepal. Currently NHE (Nepal Hydro Electric (P) Ltd) and KMI (Kathmandu Metal industry) are the major manufacturer of pico-hydro units.

PEEDA (People Energy \& Environment Development Association) has identified the low-head turbine pico-hydro units developed by NHE and KMI as having a significant potential for application all over the hill and Terai region of Nepal. It has carried out a preliminary study to examine the current situation. This included the level of interest amongst potential end-users and other relevant stakeholders in the value chain (i.e. manufacturers, installers and other service providers) for the promotion, marketing and installation of low-head type pico-hydro units in selected areas of Nepal. The preliminary study concluded that the technology was a viable alternative to the high head peltric-sets or to the solar home systems, and that the low head technology would work well throughout Nepal [5].

\subsection{Pico-hydro development}

As stated earlier, NHE and KMI are the leading pico-hydro manufacturers of Nepal. Nepal Hydro Electric (P) Ltd (NHE), in collaboration with Kathmandu University and Butwal Technical Institute (BTI), have developed over a period of several years a robust propeller turbine, with generator and electronic load controller (ELC) which forms a complete pico-hydro package. NHE has recently developed the volute cased propeller turbine which has efficiency of over $73 \%$. A typical $1.2 \mathrm{~kW}$ propeller turbine requires a flow of 85 liters/s and a height drop of 3.3 meters. Kathmandu Metal Industry has developed a 'Crosstric' Set - a pico sized version of 
the cross flow turbine. This comes as an impulse turbine and induction generator set and can produce $100 \mathrm{~W}$ to $5 \mathrm{~kW}$ power. The crosstric sets require between 5 to $20 \mathrm{~m}$ head and a flow range of 5 to 50 1/s. The table below shows the pico-hydro manufactured and installed by NHE.

\section{Design Theory}

\subsection{Axial flow turbine}

A turbine whose motion is governed by the fluid flowing perpendicular to its direction of rotation is called axial flow turbine. Both admission and exit of flow is in axial direction.

The axial turbine stage comprises a row of fixed guide vanes or nozzles (often called a stator row) and a row of moving blades or buckets (a rotor row). Fluid enters the stator with absolute velocity $c_{1}$ at angle $\alpha_{1}$ and accelerates to an absolute velocity $c_{2}$ at angle $\alpha_{2}$. All angles are measured from the axial $(x)$ direction. The sign convention is such that angles and velocities as drawn in are taken as positive. From the velocity diagram, the rotor inlet relative velocity $w_{2}$, at an angle $\mathrm{b}_{2}$, is found by subtracting, vectorially, the blade speed $U$ from the absolute velocity $c_{2}$. The relative flow within the rotor accelerates to velocity $w_{3}$ at an angle $b_{3}$ at rotor outlet; the corresponding absolute flow $\left(c_{3}, \alpha_{3}\right)$ is obtained by adding, vectorially, the blade speed $U$ to the relative velocity $w_{3}[1]$.

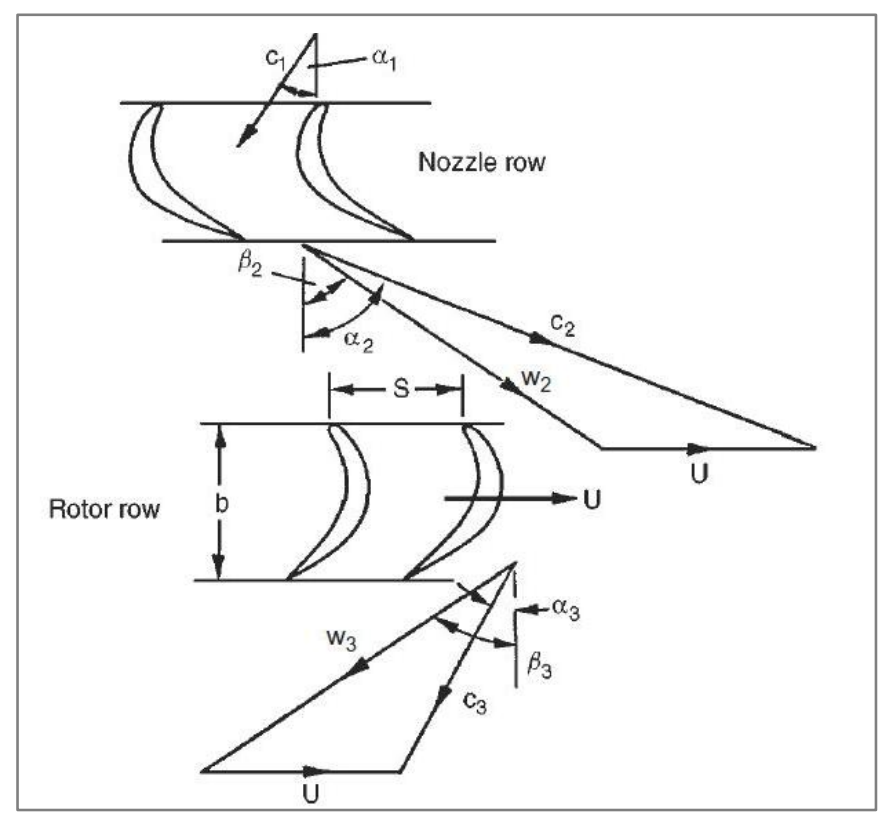

Figure 2.1: Velocity diagram for axial flow turbine (Dixon and Hall)

\subsection{Euler Turbine Equation}

Torque, $\tau$ developed in a turbine is equal to the rate of change of angular momentum of the fluid passing through the turbine. Torque is given by the product of the mass flow rate, $\dot{m} \&$ and the change in the product of radius, $r$, and circumferential fluid velocity, $C_{\theta}$, at the inlet and exit positions of the control volume surrounding the blade row. The inlet and exit are denoted by subscripts 2 and 3, respectively. For turbines a steady mass flow rate can be assumed. 


$$
\boldsymbol{\tau}=\dot{\mathbf{m}}\left(\mathbf{r}_{2} \mathbf{C}_{\theta 2}-\mathbf{r}_{3} \mathbf{C}_{\theta 3}\right)
$$

The rate of work transferred from the fluid to the runner, $\dot{E}_{t}$ is the product of torque and angular velocity, $\omega$.

$$
\dot{\mathrm{E}}_{\mathrm{t}}=\tau \omega=\omega \dot{\mathrm{m}}\left(\mathrm{r}_{2} \mathrm{C}_{\theta 2}-\mathrm{r}_{3} \mathrm{C}_{\theta 3}\right)
$$

\subsection{Free vortex theory}

Propeller turbine is classified in the category of incompressible axial flow turbines, thus the free vortex law is suitable to be use for the analysis. The origins of free vortex law come essentially from the law of conservation of angular momentum. The primary conditions like irrotational flow and constant axial velocity need to be satisfied for this law. The following equation represents the final form of the free vortex law.

$$
\mathrm{C}_{\mathrm{u}} \mathrm{r}=\text { Constant }
$$

The free vortex law calls for maintaining the product of tangential flow velocity and the radius vector constant all along the inlet region and the exit region of the blade as given by equation below.

$$
\left(\mathrm{C}_{\mathrm{u}} \mathrm{r}\right)_{\text {inlet }}=\mathrm{K}_{\text {inlet }} \text { and }\left(\mathrm{C}_{\mathrm{u}} \mathrm{r}\right)_{\text {exit }}=\mathrm{K}_{\text {exit }}
$$

The constants $\mathrm{K}_{\text {inlet }}$ and $\mathrm{K}_{\text {exit }}$ are not same in magnitude. In general for an axial flow turbine the constant $\left(\mathrm{K}_{\text {inlet }}\right)$ at the inlet depends on the hydraulic (Euler) head to be realized on the shaft. In order to maximize the energy transfer, the exit tangential velocity is taken as zero (i.e. $C_{u \text {,exit }}=0$ ) all along the exit blade profile and hence $\mathrm{K}_{\text {exit }}=0$. Further, the radius vector of the axial flow turbine increases continuously from the hub to the tip, which causes the $\mathrm{Cu}$ component to decrease. This causes fluid to enter each radial section with a different swirl angle, $\alpha$. Moreover, since every radial section has a different tangential blade velocity $(\mathrm{u})$, the blade angle (or relative flow angle, $\beta$ ) should also change from the hub to tip (refer to velocity triangles in the following figure). The same holds true for the exit blade section despite $\left(\mathrm{C}_{\mathrm{u}, \mathrm{exit}}=0\right)$.

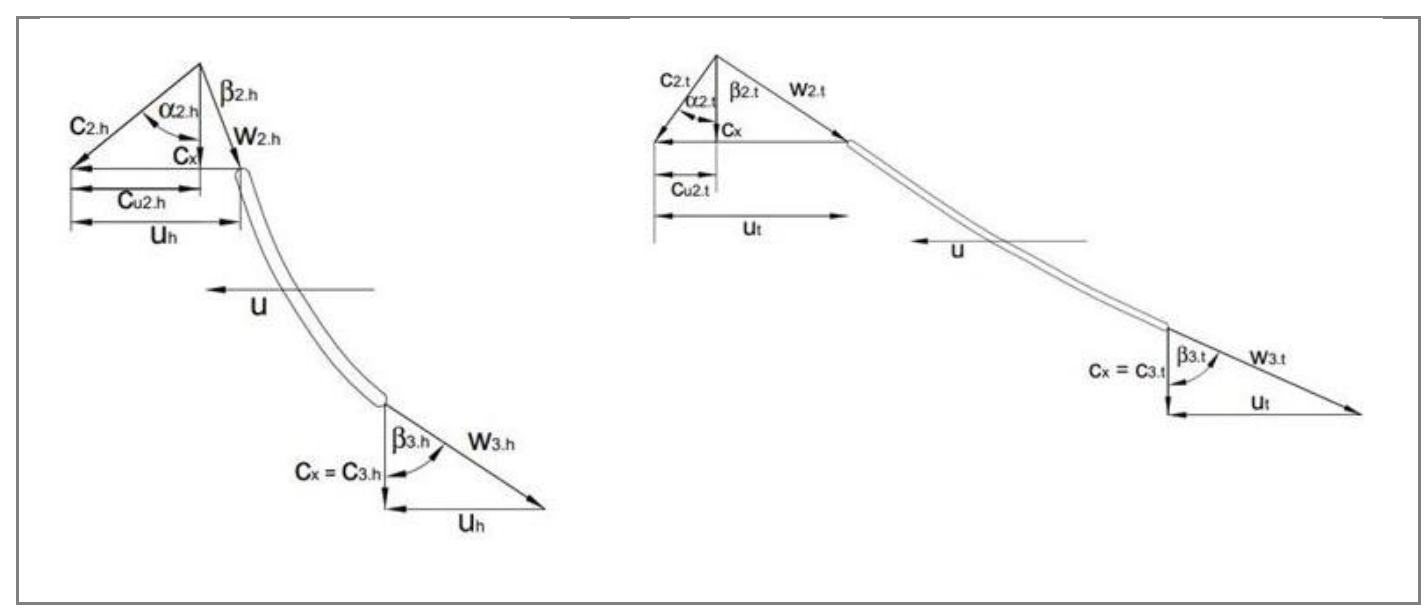

Figure 2.2: Velocity diagram for free vortex theory [7]

\subsection{Runner Design and its Parameters}


The key design parameters for a turbine are head $(\mathrm{H})$, volume flow rate $(\mathrm{Q})$ and rotational speed $(\mathrm{N})$. The choice of the speed $(\mathrm{N})$ depends on the speed of the generator and type of the drive used.

\subsection{Specific Speed and Specific Diameter}

The specific speed of a turbine, Ns, relates the flow rate through the turbine to rotational speed and head given by the relation

$$
\mathbf{N}_{\mathbf{s}}=\frac{\mathbf{N} \sqrt{\mathbf{Q}}}{(\mathrm{gH})^{0.75}}
$$

where, $\mathrm{N}$ is the rotational speed of turbine $(\mathrm{rpm}), \mathrm{Q}$ is the volume flow rate $\left(\mathrm{m}^{3} / \mathrm{s}\right), \mathrm{g}$ is the acceleration due to gravity, and $\mathrm{H}(\mathrm{m})$ is the head.

In terms of radian above equation can be written as

$$
\mathbf{\Omega}_{\mathbf{s}}=\frac{\Omega \sqrt{\mathbf{Q}}}{(\mathbf{g H})^{0.75}}
$$

Often the $\mathrm{g}$ term is dropped to make the equation dimensionless number giving the form as

$$
\mathbf{n}_{\mathbf{s}}=\frac{\mathbf{N} \sqrt{\mathbf{Q}}}{(\mathbf{H})^{0.75}}
$$

The specific speed gives an indication of the geometry of the turbine and it is the starting point for detailed design.

According to Simpsons and Williams the expected range of specific speed values is $70<\mathrm{n}_{\mathrm{s}}<300$. Specific diameter, $D_{s}$, is similar to the specific speed, but relates performance to the turbine characteristic diameter, $D$, instead of speed

$$
\mathrm{D}_{\mathrm{s}}=\frac{\mathrm{D}(\mathrm{gH})^{0.25}}{\sqrt{\mathrm{Q}}}
$$

\subsection{Hub Tip Diameter Ratio}

The hub-tip diameter ratio, $D_{h} / D_{t}$, defines hub diameter and the annulus flow area for propeller turbines. This also defines the absolute fluid velocity in the axial direction through the annulus, $C_{x}$, since flow rate, $Q$, is the product of the absolute fluid velocity in the axial direction and the annulus area [3].

Referring to Figure, which is an adapted graph based on efficient Kaplan turbine design, the tipto-head velocity ratio, $\mathrm{K}_{\mathrm{ug}}$, hub-to-tip diameter ratio, $D_{h} / D_{t}$, and the number of blades can be determined [6].

The tip-to-head velocity ratio can also be calculated as follows, resulting in the determination of tip radius, $\mathrm{r}_{\text {tip }}$

$$
\mathbf{K}_{\mathbf{u g}}=\frac{\mathbf{r}_{\mathrm{tip}} \times \boldsymbol{\omega}}{\sqrt{\mathbf{2 g H}}}
$$

where $r_{\text {tip }}$ is the blade tip radius, i.e. $r_{\text {tip }}=D / 2$ and $\omega$ is the angular velocity of the turbine runner, in $\mathrm{rad} / \mathrm{s}$, i.e. $\omega=2 \pi \mathrm{N} / 60 . \mathrm{K}_{\mathrm{ug}}$ is calculated according to the specific speed, based on the graph shown below. 


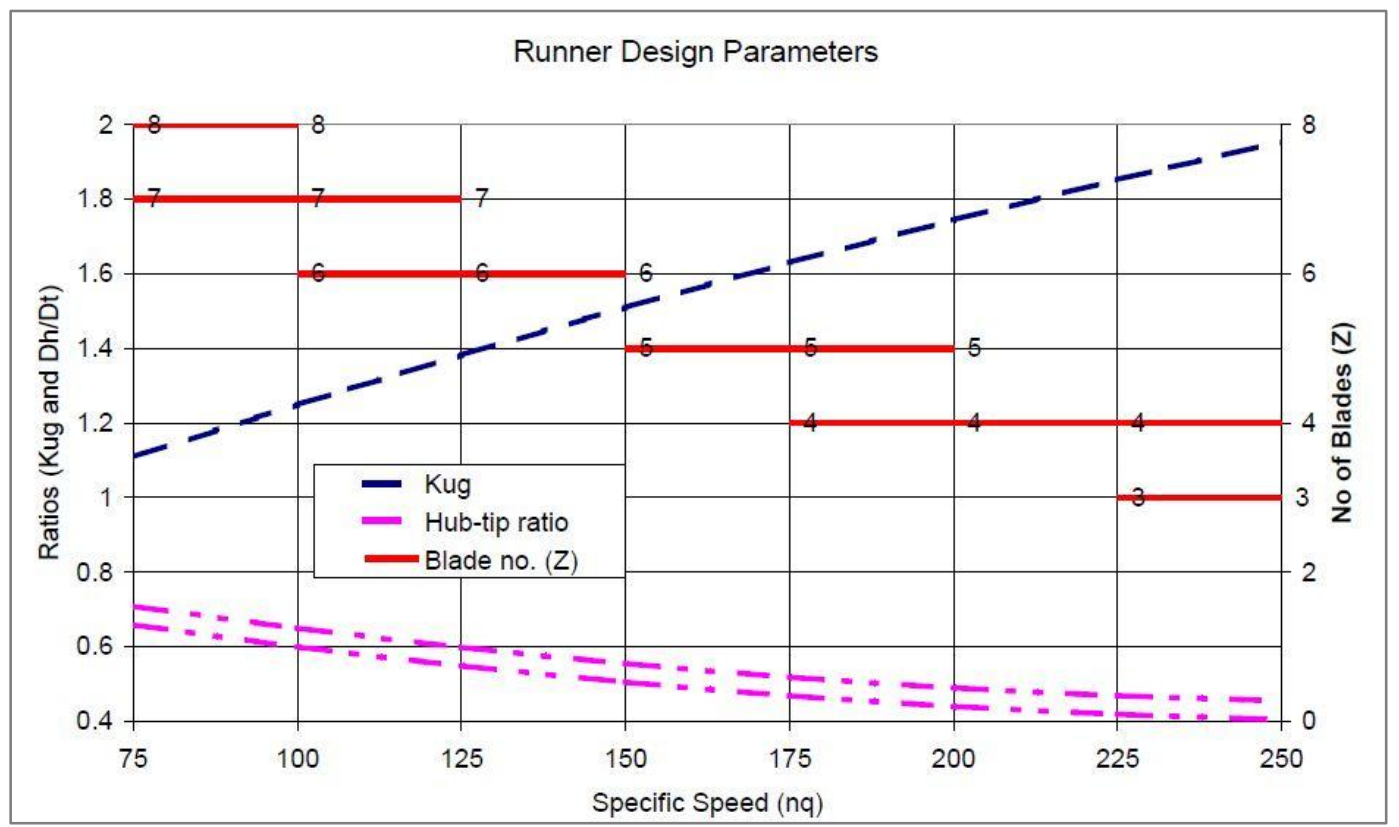

Figure 2.3: Runner Design Parameters

Wright (1999) suggests referring to the Cordier diagram which correlates specific speed and specific diameter based on empirical data of a range of different turbo machines, and can be used to determine tip diameter.

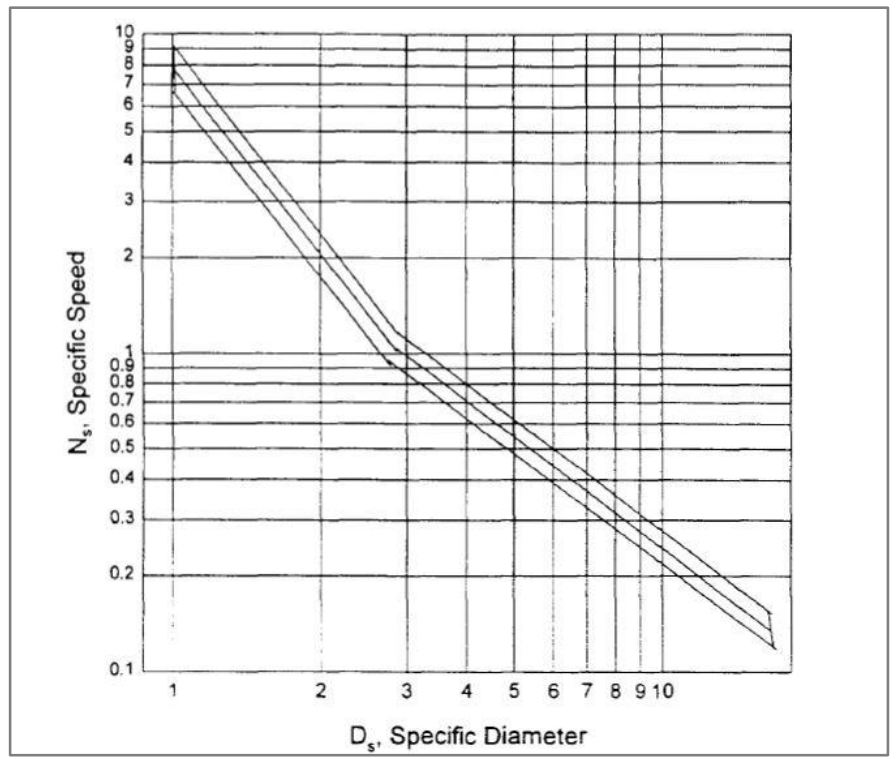

Figure 2.4: Cordier correlation of $\mathrm{N}_{\mathrm{s}}$ and $\mathrm{D}_{\mathrm{s}}[10]$ 


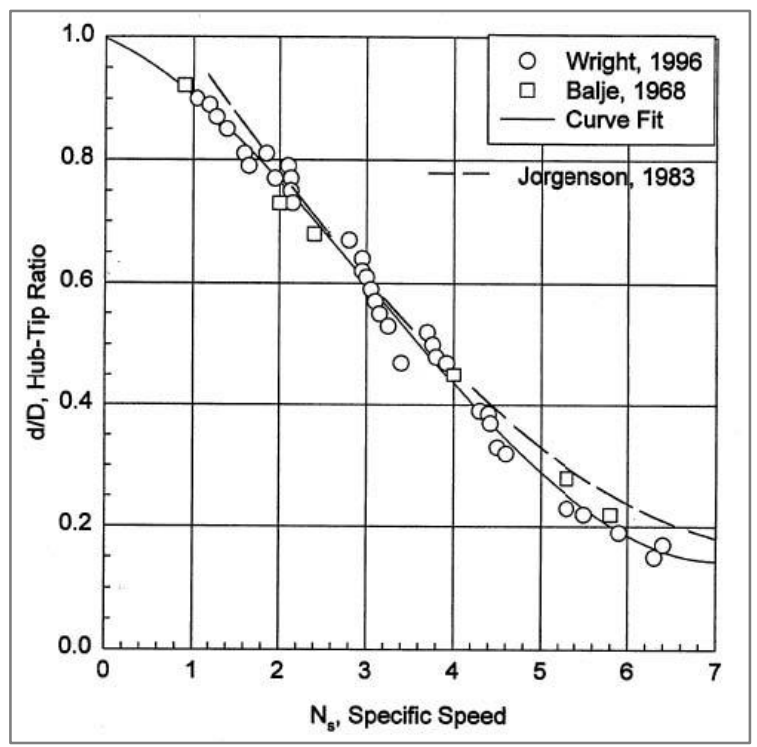

Figure 2.5: Hub-tip diameter ratio as function of specific speed using de Haller limit [10]

A curve fit applied by Wright (1999) to the data points gives following relation.

$$
\frac{D_{h}}{D_{t}}=\left(\frac{1}{2}\right)\left[1-\left(\frac{2}{\pi}\right) \tan ^{-1}\left\{\left(\frac{2}{\pi}\right)\left(N_{s}-3.8\right)\right\}\right]
$$

Applying this analysis, a hub- tip diameter can be found.

According to Singh and Nestmann, the choice of the hub to tip ratio and number of blades technically depends on the specific speed of turbine. However, a designer's freedom can also be exercised in choosing the values. They also concluded that having a larger hub-tip diameter ratio yielded lower runner losses [7].

\subsection{Velocity diagrams at different blade sections}

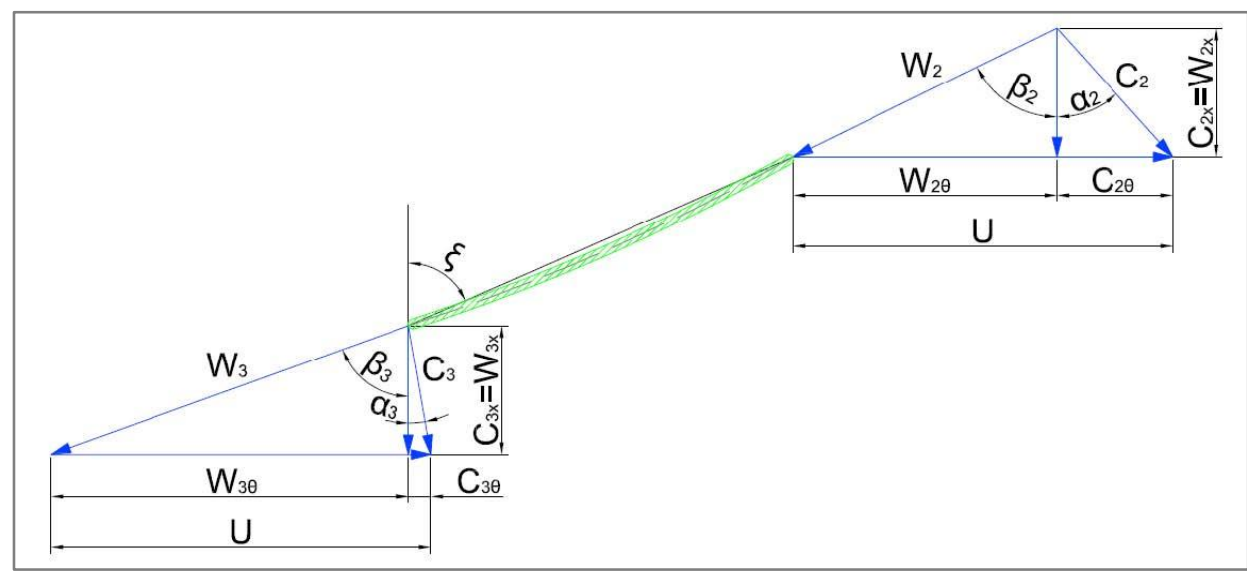

Figure 2.6: Velocity diagram (Ho-Yan,2012)

From literature survey, it is suggested that maintaining some swirl in the fluid velocity when entering the draft tube to reduce separation and increase efficiency in draft tube. Simpson and 
Williams (2011) recommend setting the relative exit velocity to the runner blade velocity i.e., $\mathrm{W}_{3}=\mathrm{U}$.

From [1], the blade stagger angle $\xi$ is the angle between blade chord line and the reference direction (axial). For circular arc blades the stagger angle is defined as

$$
\xi=\frac{1}{2}\left(\beta_{2}+\beta_{3}\right)
$$

From the velocity diagrams, the radial variation in the flow can be characterized at different sections along the radial coordinates of the blade equally spaced between the hub and tip locations along the length of the blade.

The number of blades, blade pitch, and chord length are interconnected. An increase in the number of blades, or a reduction in spacing between blades, improves flow guidance, however increases losses due to friction. Conversely, with greater blade spacing, lower flow guidance results in increased losses due to flow separation [3].

To optimize the balance, Zweifel established a criterion for the ratio of tangential loading comparing the actual ideal case. The Zweifel criterion, $\mathrm{Z}$, is defined as

$$
\mathrm{K}_{\mathbf{b}}=\frac{2 \mathrm{gH \eta}^{\mathrm{x}} / 1}{\mathrm{Uw}_{\infty}}=\mathrm{MC}_{\mathrm{L}}
$$

It is suggested that the value of $\mathrm{K}_{\mathrm{b}}$ at the hub should be approximately 0.8 [6] . The value of $\mathrm{M}$ depends on the value of $\beta \infty$ and $x / 1$.

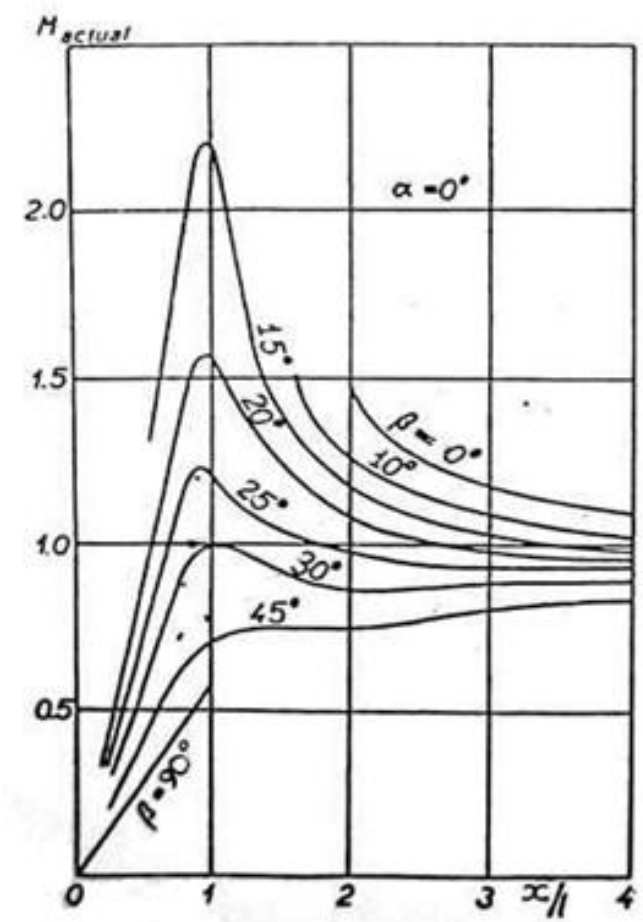

Figure 2.7: Factor to modify Lift coefficient due to multiple blades [6]

Although it is strictly for $\alpha=0$, it is applicable to other values, as long as the blades are relatively thin [4]. 
Usually the chord length (l) should increase from hub to tip, but the ratio of pitch: chord, $x / 1$, should also increase from hub to tip. Normally, the angle of attack and the blade camber will both increase from blade tip to blade hub. The values should be chosen to give the values of lift coefficient in the spreadsheet line $\mathrm{C}_{\mathrm{L}}[6]$.

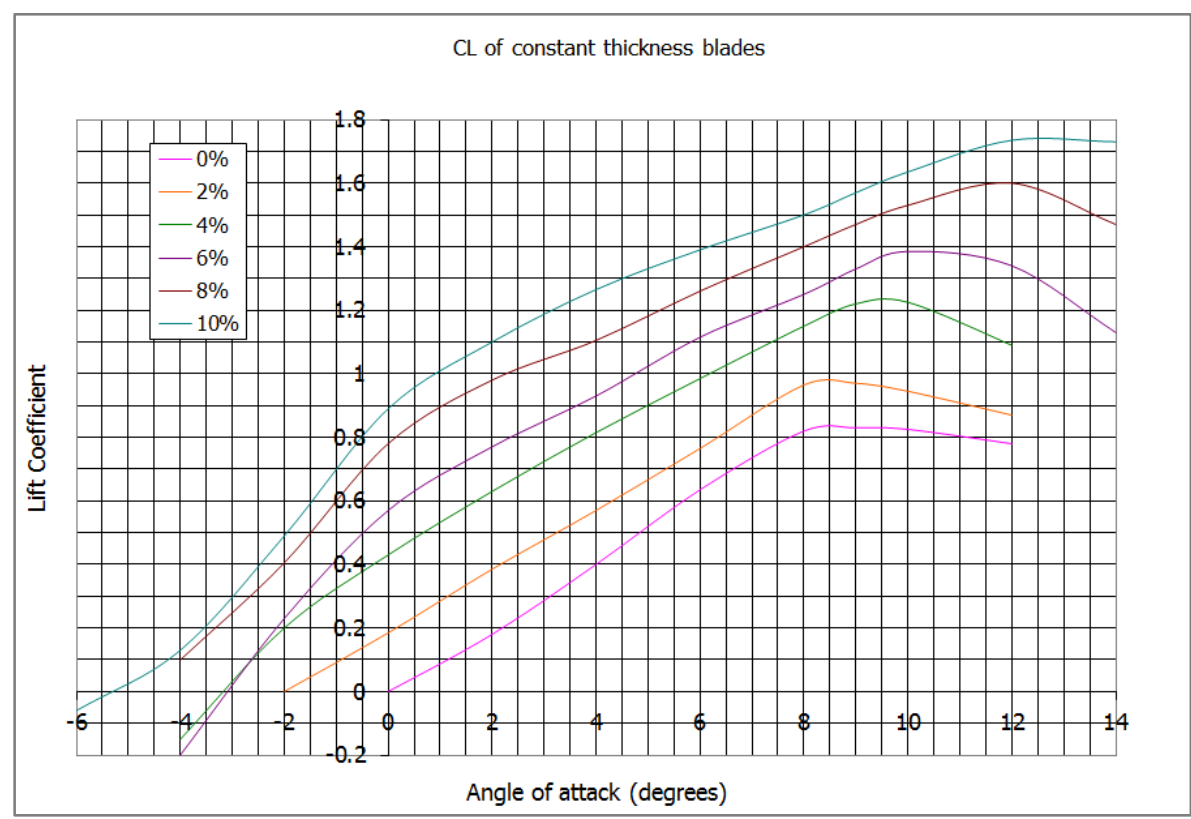

Figure 2.8: lift coefficients for thin, constant thickness blades [6]

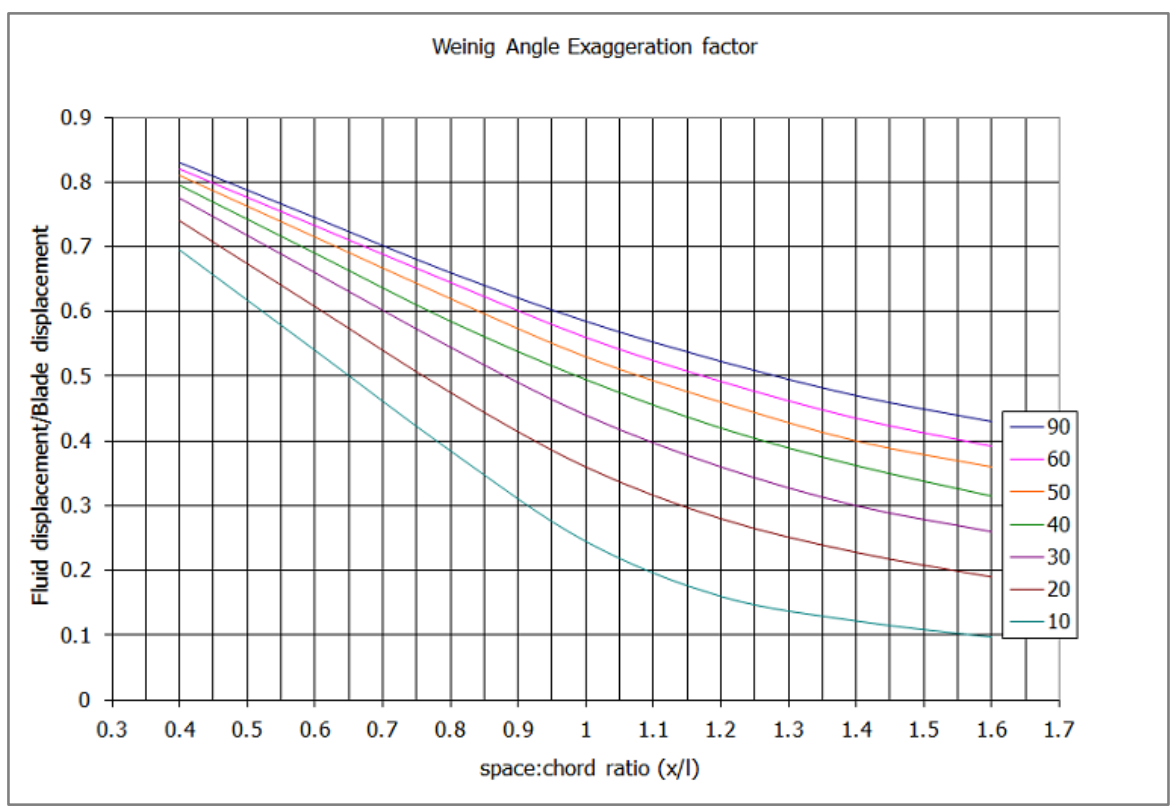

Figure 2.9: Weining Angle Exaggeration Factor [6]

An "angle exaggeration factor", $\delta$, that can be used to find the ratio of flow deflection to blade deflection, based on the work of Weining. If the actual value of "theta ratio" is greater than the 
value extracted from the chart, then the $\mathrm{C}_{\mathrm{L}}$ chart should be used again. The angle of attack should be reduced and the camber increased to give the same required value of lift coefficient, but with a greater value of blade deflection [6].

\subsection{Draft tube}

Williams (2011) suggested a flare of $4^{\circ}$ from vertical for a height of $10 D_{t}$

\subsection{Open spiral volute}

It is an open flume volute that generates swirl into the flow prior to entering the stator. For the given gross head of $2 \mathrm{~m}$, the turbine is envisaged to work under a suction head of $1.3-1.4 \mathrm{~m}$ and an inlet head with $0.4-0.6 \mathrm{~m}$, which eliminates the need of a closed spiral volute inlet. The volutes of other propeller researchers are closed type since their runners operate at a higher inlet head [7].

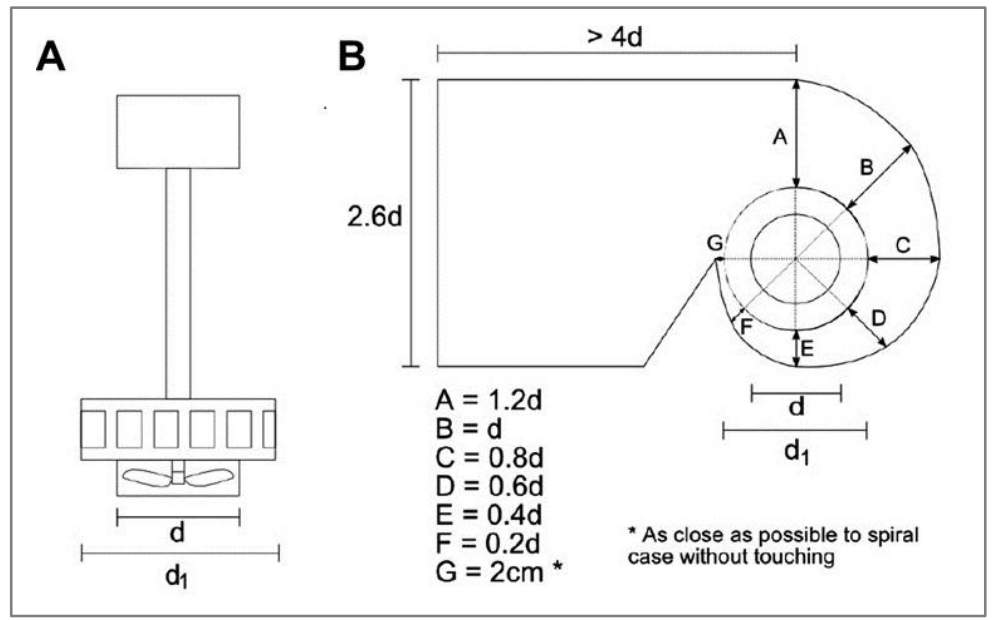

Figure 2.10: Guidelines for open spiral volute casing (Susanto \& Stamp, 2012)

The design is based on the specifications provided by J. Susanto and S. Stamp.The open spiral volute has been adapted by [7], [3], and by Asian Phoenix Resources Ltd. in their Powerpal turbines.

\subsection{Guide vanes}

Guide vane allows radial inflow through a set of guide vanes to further generate swirl in the flow. The guide vanes are straight blades set in a circular array around the rotation axis. Straight blades are preferred for the ease of manufacture. After exiting the guide vanes, the flow is directed downward parallel to the axial direction towards the runner, while contained within cylindrical tube housing.

$$
\begin{aligned}
& h_{G V}=0.4 D_{t} \\
& \lambda=0.25 D_{t} \\
& \alpha_{G V}=\tan ^{-1} \frac{C_{2 \theta}}{Q / 2 \pi r_{G V} h_{G V}}
\end{aligned}
$$


where $C_{2 \theta}$ the tangential fluid velocity at the runner inlet and $\mathrm{Q}$ is is the flow rate. The tangential flow corresponding to the mid blade station is used to determine the angle setting.

$$
n=\frac{1}{4} \sqrt{D}+(4 \text { to } 6)
$$

Where, $\mathrm{n}$ is the number of vanes and $\mathrm{D}$ is runner tube diameter.

\section{Model Design}

\subsection{Scaling laws}

If a model and prototype are "similar", i.e., their shape is the same (at a different scale) and the flow patterns (streamlines) are the same throughout, then they will have the same efficiency at any particular operating point. The scale ratio of the size of the turbines is determined using a typical dimension, which for turbines is usually the blade tip diameter (D). To get "similar" flow Reynolds's number ( $\mathrm{Re})$ should be equal for the model and prototype. However it is generally accepted that for turbulent flow (i.e., large Re) the flows will be sufficiently similar even if $R e$ is not equal.

$$
\operatorname{Re}=\frac{\rho \omega \mathrm{D}^{2}}{\mu}
$$

As well as changing the size of the prototype, the head can also be changed. For the prototype to be at the same operating point as the model, the axial velocity of the flow must remain in proportion to the tangential velocity of the blades. This gives the same flow angles relative to the blades and hence the same flow patterns, so that similarity is maintained [2].

Power is a function of flow rate $(\mathrm{Q})$, specific energy rise $\mathrm{gH}$, machine size $(\mathrm{D})$, rotational speed $(\omega)$ and liquid properties $\mu, \rho$ and $\kappa$ (modulus). Using the principle of dimensional analysis sets of non dimensional groups may be obtained such as:

$$
\frac{P}{\rho \omega^{3} D^{5}}=f\left[\frac{Q}{\omega D^{3}}, \frac{g H}{\omega^{2} D^{2}}, \frac{\rho \omega D^{2}}{\mu}, \frac{\rho \omega^{2} D^{2}}{\kappa}\right]
$$

Where, $\quad \frac{P}{\rho \omega^{3} D^{5}}$ is power coefficient

$$
\begin{aligned}
& \frac{Q}{\omega D^{3}} \text { is flow coefficient } \\
& \frac{g H}{\omega^{2} D^{2}} \text { is head coefficient } \\
& \frac{\rho \omega D^{2}}{\mu} \text { Reynolds number based on a typical machine design } \\
& \frac{\rho \omega^{2} D^{2}}{\kappa} \text { form of Mach number }
\end{aligned}
$$

The first three groups may be used to predict the probable dynamically similar performance of the same machine running at different speeds, or of a machine in the same family using scaling laws. (Turton, 1995) 


$$
\begin{aligned}
& \frac{P}{\rho \omega^{3} D^{5}}=\text { const } \\
& \frac{Q}{\omega D^{3}}=\text { const } \\
& \frac{g H}{\omega^{2} D^{2}}=\text { const }
\end{aligned}
$$

This requires that the flow patterns in each must be the same and that they are the same shape, i.e., have a consistent scale ratio throughout. The size of the turbine is given by a characteristic dimension, which in this case is $\mathrm{D}$, the blade tip diameter. The scaling laws can be arranged into various forms as

$$
\begin{aligned}
& \frac{Q_{p}}{Q_{m}}=\left(\frac{H_{p}}{H_{m}}\right)^{1 / 2}\left(\frac{D_{p}}{D_{m}}\right)^{2} \\
& \frac{N_{p}}{N_{m}}=\left(\frac{H_{p}}{H_{m}}\right)^{1 / 2}\left(\frac{D_{p}}{D_{m}}\right)^{-1} \\
& \frac{P_{p}}{P_{m}}=\left(\frac{H_{p}}{H_{m}}\right)^{3 / 2}\left(\frac{D_{p}}{D_{m}}\right)^{2}
\end{aligned}
$$

The use of models for predicting full size water turbine performance is well established. Significant differences in efficiency between model and full size occur, suggesting departures from strict dynamic similarity. Departures from the scaling laws are due to:

- Geometrical dissimilarities due to tolerances

- Clearance variations

- Surface finish

- Hydrodynamic effects

- Testing errors

- Installation effects

It is argued by both turbine and pump authorities that losses differ, and that work/capacity curves differ too. All are agreed that exact mathematical treatment is not possible, and attempt empirical formulation from experimental data. One of such formulas is the turbine equation by Hutton which is expressed as,

$$
\frac{1-\eta_{\mathrm{p}}}{1-\eta_{\mathrm{m}}}=0.3+0.7\left(\frac{\mathrm{Re}_{\mathrm{m}}}{\mathrm{Re}_{\mathrm{p}}}\right)^{0.2}
$$

[9]

For the model turbine development, the flow rate and head was fixed to $251 / \mathrm{s}$ and $1 \mathrm{~m}$ to ascertain with the specification of IWM test rig. The scale ratio was found to be $27 / 38$ and it was used to calculate the parameters of other components like guide vanes, spiral volute, draft tube etc. 


\section{Design Summary}

The summary of design for prototype and model are tabulated as follows.

\begin{tabular}{|c|c|c|c|c|}
\hline \multicolumn{3}{|c|}{ Summary of Design } & Prototype & Model \\
\hline \multirow[t]{9}{*}{1} & \multicolumn{4}{|c|}{ Runner Parameters } \\
\hline & 1.1 & Speed of runner N (rpm) & 1000 & 995 \\
\hline & 1.2 & Tip Diameter $\mathrm{D}_{\text {tip }}(\mathrm{mm})$ & 190 & 135 \\
\hline & 1.3 & Hub Diameter $\mathrm{D}_{\text {hub }}(\mathrm{mm})$ & 114 & 80 \\
\hline & 1.4 & No of Blades (n) & 5 & 5 \\
\hline & 1.5 & Aligned chord length at tip (mm) & 105 & 75 \\
\hline & 1.6 & Aligned chord length at mid (mm) & 95 & 67.5 \\
\hline & 1.7 & Aligned chord length at hub (mm) & 85 & 60.4 \\
\hline & 1.8 & Material of runner & High Carbon Steel & Mild Steel \\
\hline \multirow[t]{5}{*}{2} & \multicolumn{4}{|c|}{ Shaft } \\
\hline & 2.1 & Diameter of shaft D (mm) & 40 & 25 \\
\hline & 2.2 & Length of shaft between bearings L(mm) & 800 & 350 \\
\hline & 2.3 & Key size $(\mathrm{mm})$ & & $8 \mathrm{~mm}$ dia \\
\hline & 2.4 & Material & Cold Rolled Steel & Mild Steel \\
\hline 3 & \multicolumn{4}{|c|}{ Bearing Specification } \\
\hline & 3.1 & Bearing & NU2208 (SKF) & NU 2205 (SKF) \\
\hline 4 & \multicolumn{4}{|c|}{ Draft tube } \\
\hline & 4.1 & Flare angle & $4^{\circ}$ & $4^{\circ}$ \\
\hline & 4.2 & Height $(\mathrm{mm})$ & 1900 & 1350 \\
\hline & 4.3 & Inlet diameter $(\mathrm{mm})$ & 200 & 142 \\
\hline & 4.4 & Outlet Diameter (mm) & 465 & 286 \\
\hline \multirow[t]{7}{*}{5} & \multicolumn{4}{|c|}{ Guide Vanes } \\
\hline & 5.1 & Inner Diameter (mm) & 200 & 142 \\
\hline & 5.2 & Outer Diameter (mm) & 300 & 213 \\
\hline & 5.3 & Height of vanes (mm) & 76 & 54 \\
\hline & 5.4 & Setting angle $\left({ }^{\circ}\right)$ & $71^{\circ}$ & $77^{\circ}$ \\
\hline & 5.5 & No of vanes $(n)$ & 9 & 8 \\
\hline & 5.6 & Position of runner from stator base $\lambda(\mathrm{mm})$ & 47.5 & 34 \\
\hline 6 & \multicolumn{4}{|c|}{ Open Spiral Volute (Refer Fig 20) } \\
\hline & 6.1 & $\mathrm{~A}(\mathrm{~mm})$ & 240 & 170.4 \\
\hline & 6.2 & $\mathrm{~B}(\mathrm{~mm})$ & 200 & 142 \\
\hline & 6.3 & $\mathrm{C}(\mathrm{mm})$ & 160 & 113.6 \\
\hline & 6.4 & $\mathrm{D}(\mathrm{mm})$ & 120 & 85.2 \\
\hline & 6.5 & $\mathrm{E}(\mathrm{mm})$ & 80 & 56.8 \\
\hline & 6.6 & $\mathrm{~F}(\mathrm{~mm})$ & 40 & 28.4 \\
\hline & 6.7 & $\mathrm{G}(\mathrm{mm})$ & 20 & 20 \\
\hline & 6.8 & Height $(\mathrm{mm})$ & 600 & 300 \\
\hline & 6.9 & Inlet Width (mm) & 520 & 369.2 \\
\hline & 6.1 & Orifice Diameter (mm) & 200 & 142 \\
\hline
\end{tabular}




\subsection{Discussion}

The torque was measured using mechanical brake and spring balance. A flywheel was welded to the shaft and a braking system was fitted. At the end of the brake arm, the spring balance was attached to the load. The radius of the brake arm was $220 \mathrm{~mm}$.

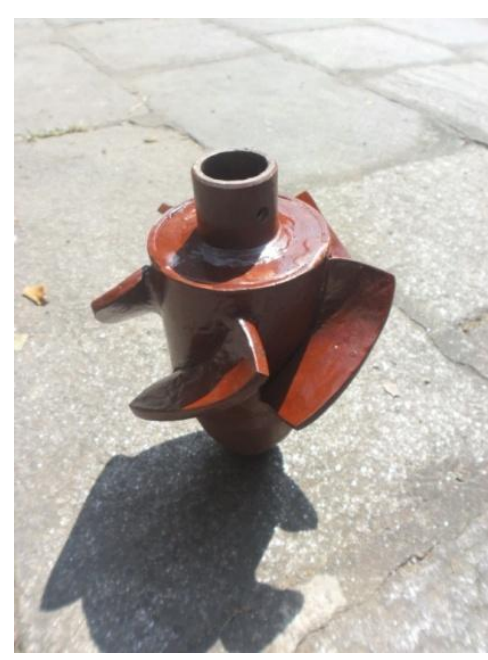

Figure 4.1: Braking system

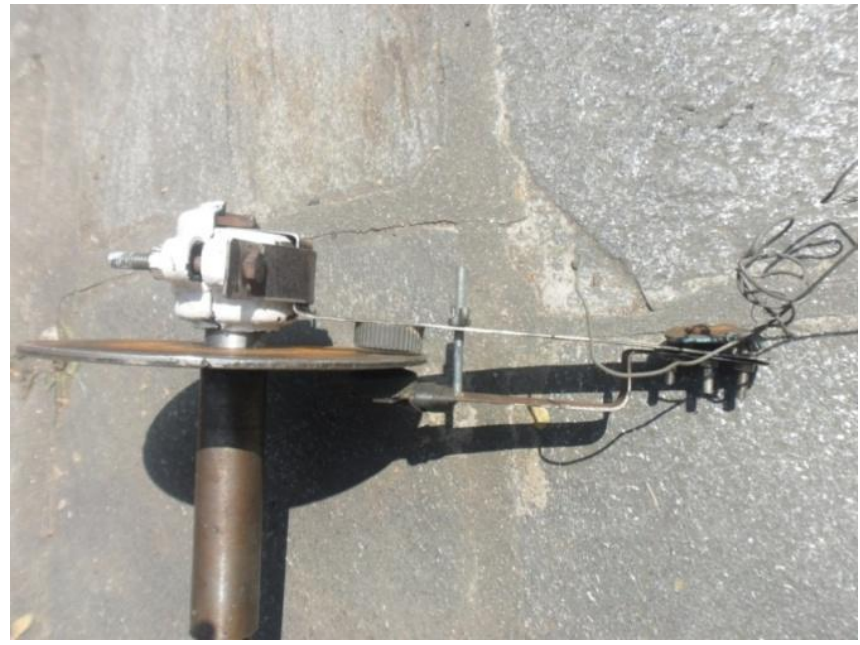

Figure 4.2: Fabricated propeller runner

For the measurement of the torque, the brake was partially applied to fix the shaft speed to certain rpm. The corresponding load was read from the spring balance. The test was carried out for three different flow rates and repeated until consistent measurements were obtained. The results obtained from the test are represented by graphs as follows:

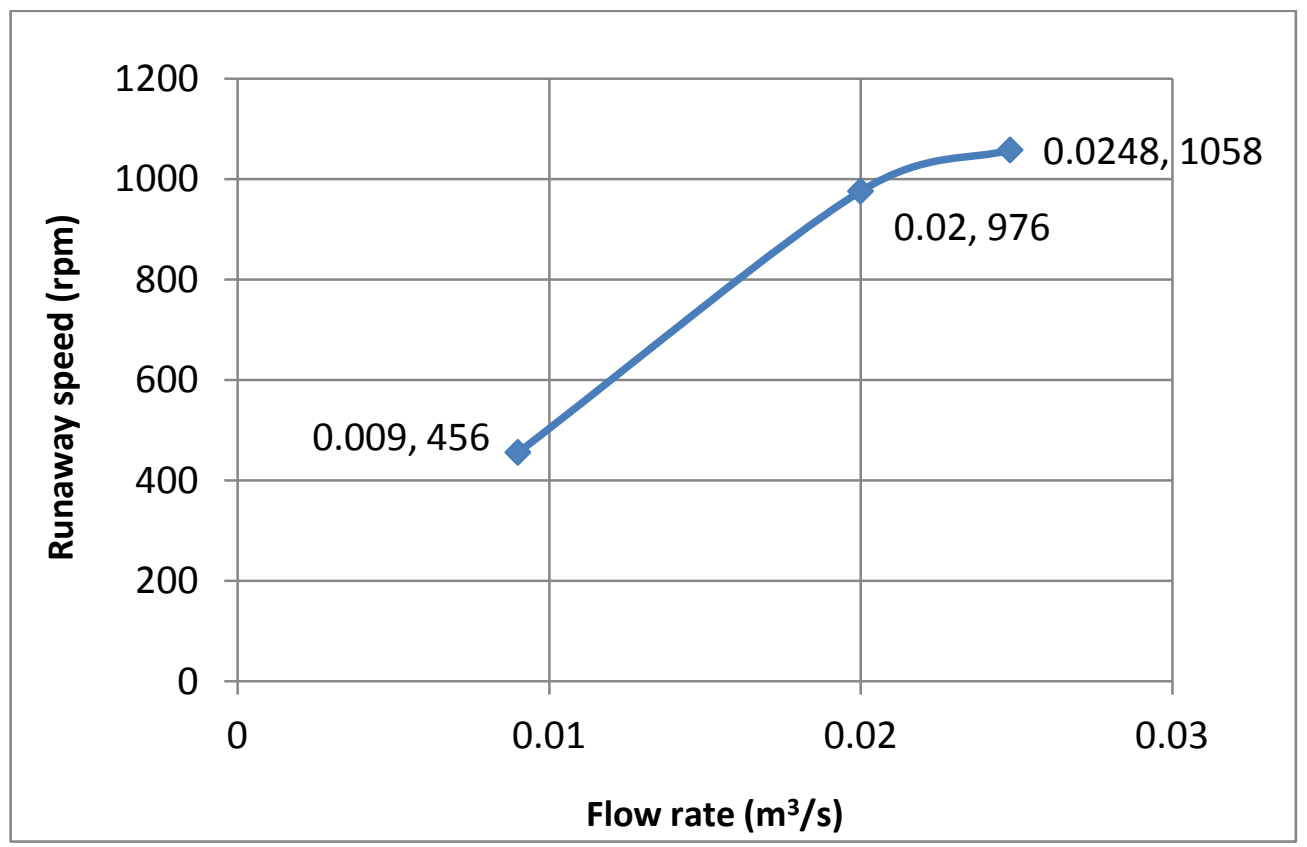

Figure 4.3: Runaway speed Vs Flow rate 
The chart above shows the runaway speed of turbine with no load applied. At design flow rate, the turbine exceeds the design speed of $1000 \mathrm{rpm}$ by $58 \mathrm{rpm}$. Also, speed increases gradually with the corresponding increase of flow rate.

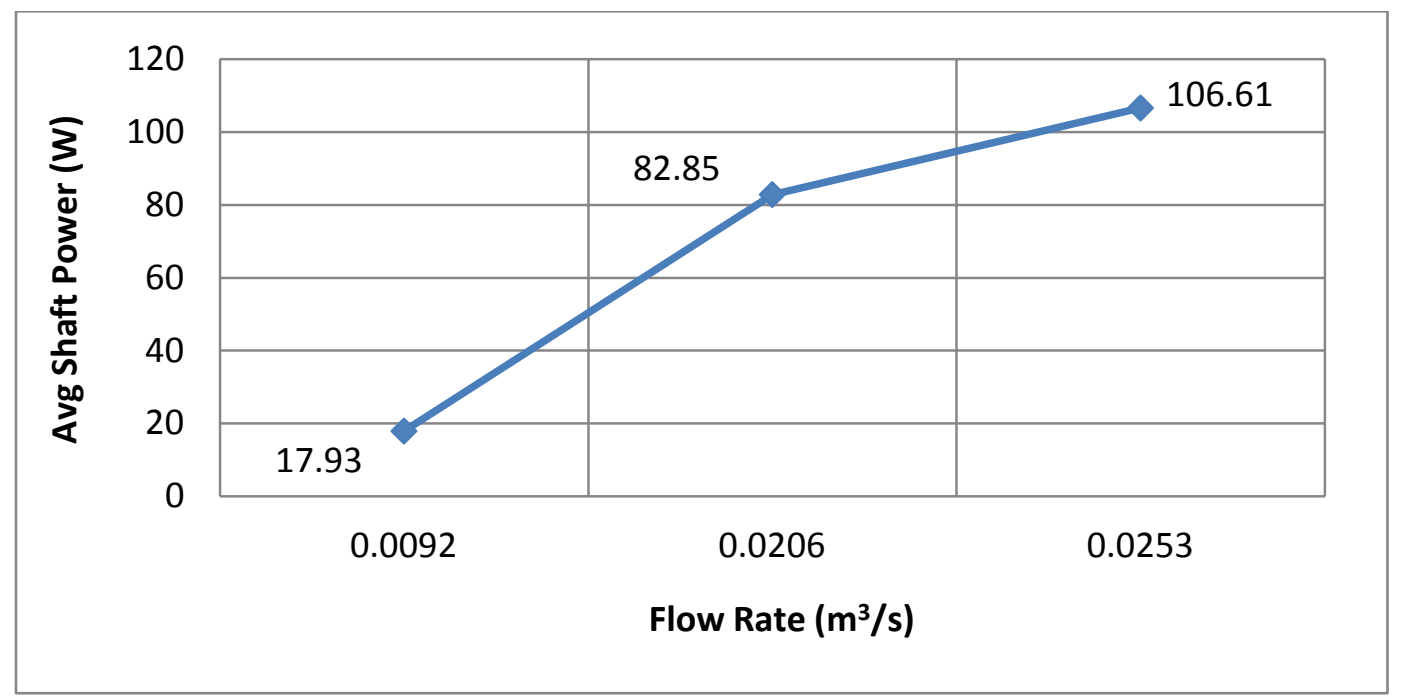

Figure 4.4: Shaft Power Vs Flow rate

The result above depicts the variation of shaft power with varying flow rates on application of load. The load applied though is not constant in each case. But, in general, with the increase of the flow rate, shaft power show the increase from $17.93 \mathrm{~W}$ at $9.2 \mathrm{l} / \mathrm{s}$ flow rate to $106.61 \mathrm{~W}$ at 25.3 $1 / \mathrm{s}$ flow rate.

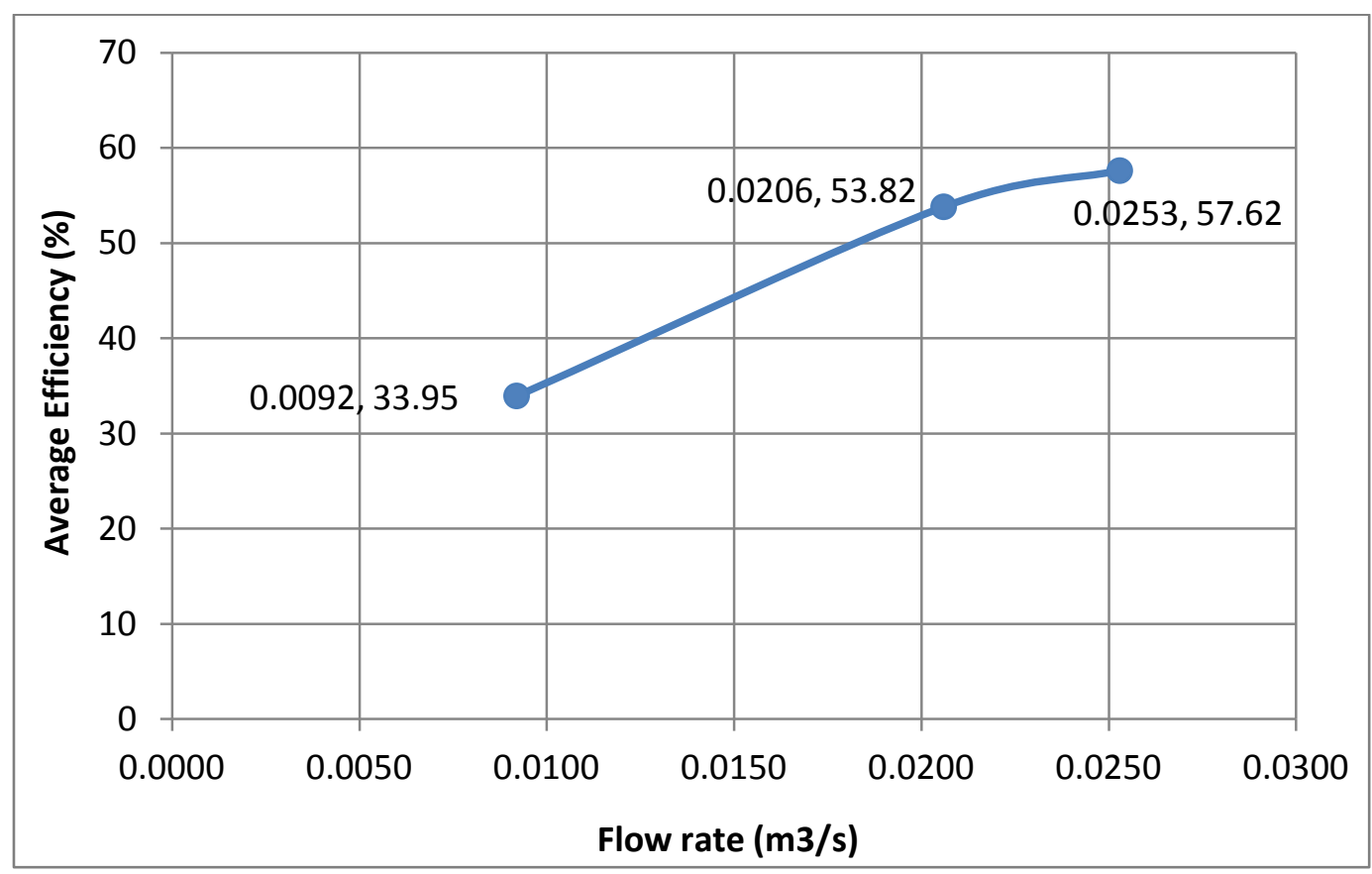

Figure 4.5: Efficiency vs. Flow Rate 
The average of the efficiencies of five tests for each flow rate was plotted against the flow rates and the trend was obtained as shown in the above chart. The efficiency of the turbine is best at full load and reduces significantly at part loads.

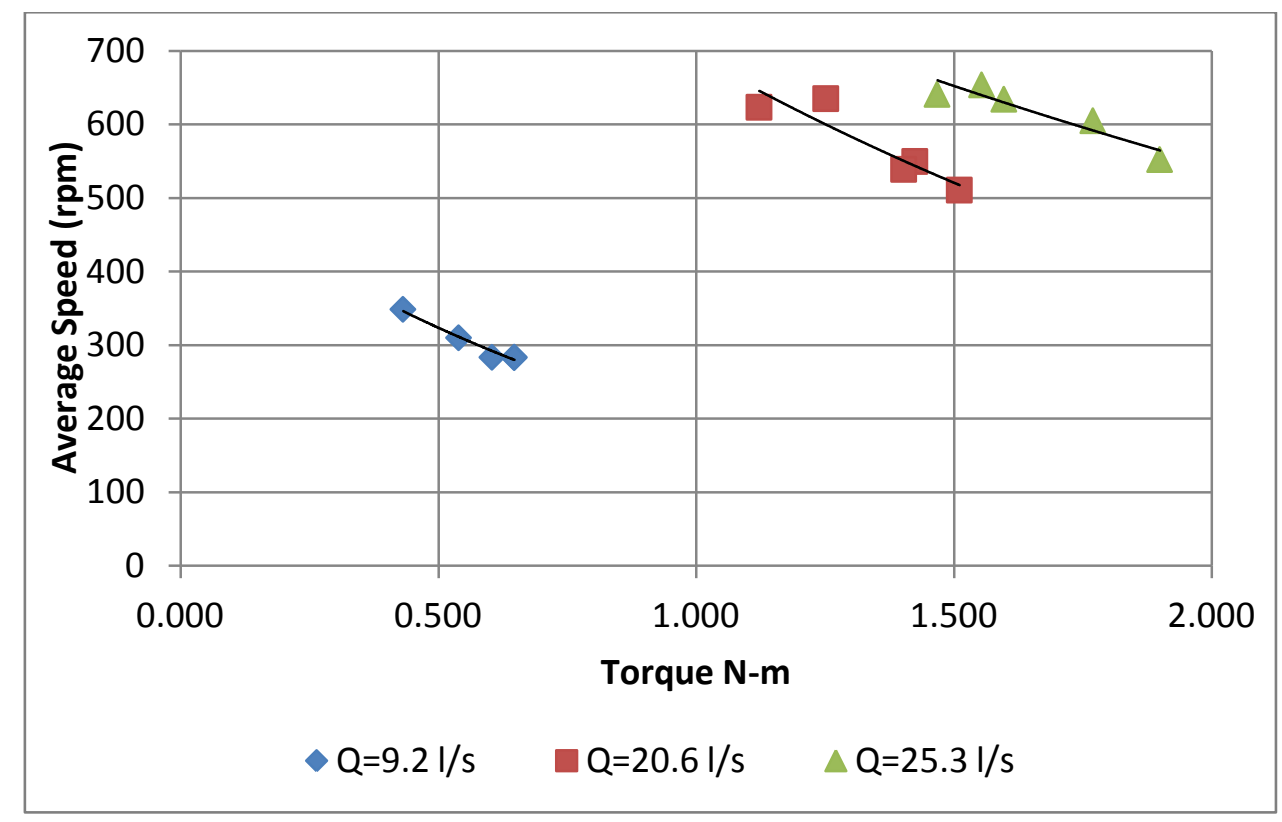

Figure 4.7: Speed Vs torque at various flow rates

The inverse relationship between speed and torque at given flow rate can be observed from the above chart. As the torque is load is increased and thus the torque, the speed of the runner decreases to balance the power output.

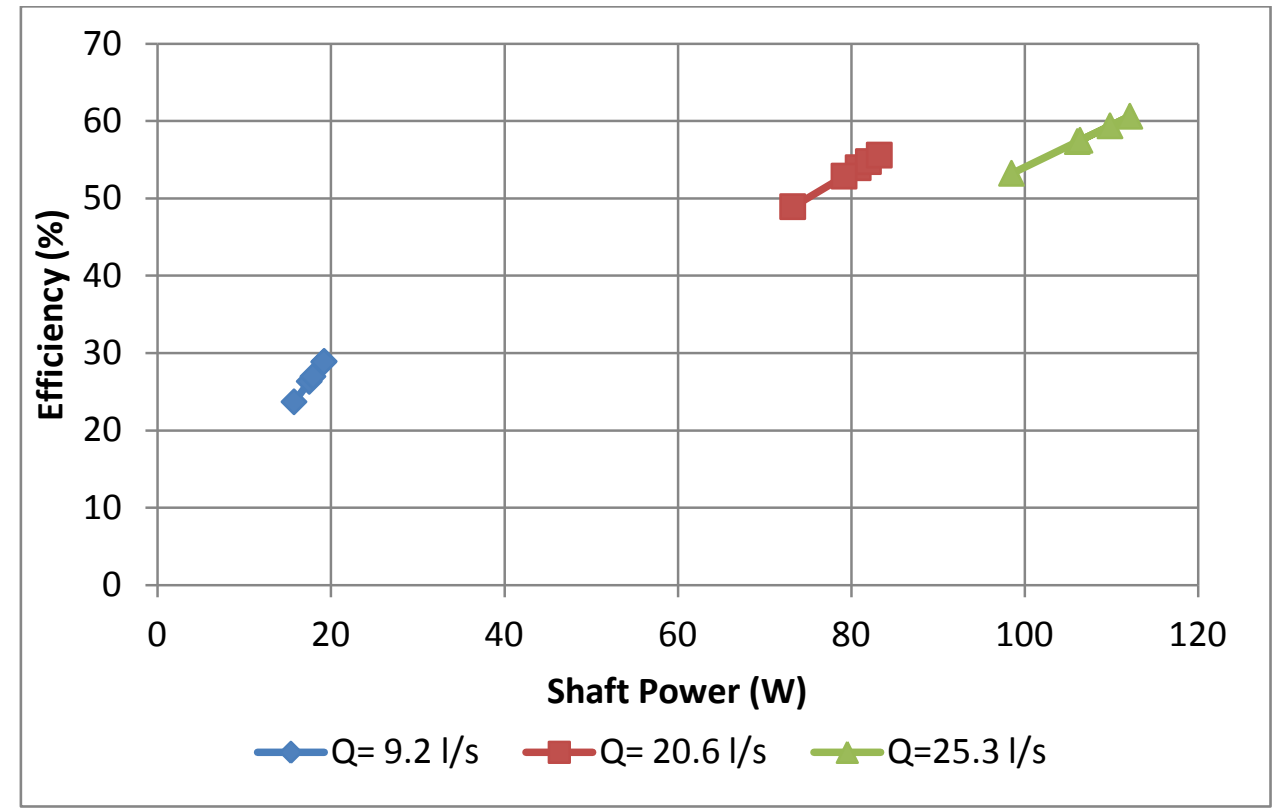

Figure 4.8: Efficiency Vs Shaft power at various flow rates 
The chart above shows the variation of shaft power and efficiency with varying flow rates. As before, the efficiency is quite low at part flow conditions but reach the maximum when design conditions are met.

\section{Conclusion}

Pico hydro can be a suitable solution to improving rural electrification and has been applied only in a few instances for medium to high head applications in Nepal. Local manufacture of pico hydro turbines has proven to be successful in other developing countries and may have led to the wide adoption of the technology. A propeller turbine with a runner with constant thickness, curved, twisted, and variable chord length blades was designed to enable $1 \mathrm{~kW}$ electrical generation with an operating head of $2 \mathrm{~m}$ and $70 \mathrm{l} / \mathrm{s}$. There are numerous sites fulfilling the condition matching for the pico hydro installation in the low lying regions of Nepal.

A scale down model operating at $25 \mathrm{l} / \mathrm{s}$ discharge and $1 \mathrm{~m}$ head was developed to match the testing condition of IWM test rig. At runaway condition, the turbine achieved the average speed of $1058 \mathrm{rpm}$ at design flow rate. At reduced flow rates $9 \mathrm{l} / \mathrm{s}$ and $20 \mathrm{l} / \mathrm{s}$, the speed was reduced to $456 \mathrm{rpm}$ and $976 \mathrm{rpm}$ respectively. On the application of load, the efficiency obtained were $33.95 \%$ and 53.82 at part flow of $9.21 / \mathrm{s}$ and $20.6 \mathrm{l} / \mathrm{s}$. The efficiency was highest at design condition with $57.62 \%$ at $25.3 \mathrm{l} / \mathrm{s}$. The efficiency however was lower than expected which can be credited to inept manufacturing processes and adversities posed by testing conditions.

Low power outputs at part load and efficiencies resulted since propeller turbine performance steeply declines during off-design operation. But as expected, the design flow rate can be achieved throughout the year in the low lying regions of Nepal, so the system can always meet the design criterion of the flow.

\section{Recommendations}

Following are the recommendations for further research and development relating to the manufacture of pico hydro turbines in Nepal and specifically to the design of a propeller turbine for low head applications.

Further testing should be carried out to assess the turbine performance when connected to electrical load.

A separate hydraulic bench with data acquisition unit is required for effective testing and development.

For the optimization of the turbine, numerous tests should be conducted by varying stagger angle, number of blades, camber and twist. Although, this process may be costly and time consuming, the design data obtained can be useful for the future references.

Airfoil blades are more efficient than flat and constant thickness blades so should be preferred for larger and efficient installations. However, for pico hydro power flat blade may prove to be cost effective.

The flow conditions may be optimized by using curved blades instead of flat blades in guide vanes. Further the effect of airfoil sections can be studied. 
To assess the real condition performance of the turbine, the system should be installed in real sites and conditions.

This system is recommended to be installed in the existing hydropower systems to generate additional electricity from tailrace water as standalone applications for the generation of electricity.

To ascertain the potential and feasibility of the pico hydro installations, a complete and detailed field study is felt necessary.

The flow conditions may be optimized by using curved blades instead of flat blades in guide vanes. Further, the effect of airfoil sections can be studied.

To assess the real condition performance of the turbine, the system should be installed in real sites and conditions.

To ascertain the potential and feasibility of the pico hydro installations, a complete and detailed field study is felt necessary.

\section{Acknowledgements}

We are highly indebted to Raj Kumar Chaulagain and Ramesh Chaudhary for their support and help in design and fabrication. Also we are thankful to all those who directly and indirectly helped us to make this project successful. We also acknowledge the receipt of support both technical and financial from "appear" project-42 (Austrian Partneship Programme in Higher Education and Research for Development) implemented by Centre for Energy Studies, Institute of Engineering, Tribhuvan University, Nepal.

\section{References}

[1] Dixon, S. L. \& Hall, C. A. (2010). Fluid Mechanics and Thermodynamics of Turbomachinery. Elsevier.

[2] Faulkner, S. A. (1991). A simplified low head propeller turbine for micro hydroelectric power, Masters Degree Thesis, University of Canterbury

[3] Ho-Yan, B. P. (2012). Design of a Low Head Pico Hydro Turbine for Rural Electrification in Cameroon, Masters Degree Thesis, The University of Guelph Guelph, Ontario, Canada

[4] Nechleba, M. (1957). Hydraulic Turbines, Their Design and Equipment, Artia Prague, Constable and Company Ltd.

[5] PEEDA. (2009). Low Head Pico-Hydro Promotion Project, Nepal, People, Energy and Environment Development Association

[6] Simpson, R. \& Williams, A. (2011). Design of propeller turbines for pico hydro, Retrieved June 15, 2012, from www.picohydro.org.uk

[7] Singh, P. \& Nestmann, F. (2009). Experimental optimisation of free vortex propeller runner, Retrieved May 12, 2012, from www.sciencedirect.com

[8] Susanto, J. \& Stamp, S. (2012). Local installation methods for low head pico-hydropower in the Lao PDR, Renewable Energy, 11, (2012) 139-117

[9] Turton, R. K. (1995). Principles of Turbomachinery, Chapmann \& Hall (pp. 29-38)

[10] Wright, T. (1999). Fluid Machinery : Performance, Analysis and Design, CRC Press 